\title{
Tendencias de utilización de sistemas de representación del conocimiento en las bibliotecas públicas y universitarias españolas
}

\author{
Trends in the use of knowledge representation systems in Spanishs public and academic libraries
}

\section{Blanca Gil Urdiciain (1), ANa Belén Ríos Hilario (2)}

(1) Departamento de Biblioteconomía y Documentación, Universidad Complutense de Madrid, mbgil@pdi.ucm.es. (2) Departamento de Biblioteconomía y Documentación, Universidad de Salamanca, anarihi@usal.es

\begin{abstract}
Resumen
Se presentan los resultados de una encuesta realizada en bibliotecas españolas, con objeto de conocer los sistemas de representación del conocimiento que emplean para la normalización de la documentación, así como los cambios que se han percibido con la llegada de la era digital. La metodología utilizada para la recopilación de los datos se basó en la solicitud de información, a través de un cuestionario, a los responsables de los diferentes centros encuestados. Entre los resultados, destacamos el bajo porcentaje de respuesta por parte de las bibliotecas consultadas. Asimismo, presentamos por un lado los datos relativos a los sistemas de representación utilizados por las diferentes bibliotecas y, por otro, se reflejan los aspectos que se han visto afectados por la era digital, en opinión de los encuestados. Las conclusiones refieren notables cambios en relación con la creciente tendencia al uso de los documentos digitales, mientras que en lo que al control del vocabulario se refiere, la situación permanece inalterada en la mayoría de las instituciones.
\end{abstract}

Palabras clave: Representación del conocimiento. Sistemas de clasificación bibliotecaria. Tesauros. Listas de encabezamientos de materias. Bibliotecas. España.

\section{Introducción}

El presente trabajo se realizó en el marco del proyecto de investigación I+D+i titulado "La nueva ecología de la información y la documentación en la sociedad del conocimiento: desarrollo de una métrica sistémica, planificación de un observatorio para su seguimiento e identificación de tendencias básicas y retos estratégicos" (1). Dicho proyecto tiene como propósito fundamental "construir un cuadro sistemático que permita dibujar claramente las características y evolución del sector en España, ampliando la discusión en colaboración con los agentes existentes" (2009, p. 6).

Los resultados que aquí se presentan corresponden concretamente a los conseguidos por el grupo "Sistemas de representación y organiza-

\begin{abstract}
We present the results of a survey carried out in Spanish libraries. Its objective was to find out which systems of knowledge representation are used to normalize documents, and what changes have been noticed since the arrival of the digital age. The data compilation method was based on information requests. A questionnaire was sent out to the persons in charge of the various establishments that were polled. Among the results, we would highlight the low percentage of response that was obtained. On the one hand, we give an account of the data referring to the knowledge representation systems used at the various libraries. On the other hand, we present the aspects which, according to the respondents, have been affected by the advent of the digital age. The conclusions report noticeable changes related to the increasing use of digital documents. However, with regards to vocabulary control, most of the institutions show no evidence of any changes.
\end{abstract}

Keywords: Library classifications. Thesauri. Subject headings. Libraries. Knowledge organization. Spain.

ción del conocimiento", cuyo doble objetivo es: 1) recopilar la normativa desarrollada en el ámbito de la Información y Documentación y, más concretamente, el análisis de las normas correspondientes al área de la representación y recuperación de la información; y 2) conocer los sistemas de representación del conocimiento que se utilizan en distintas bibliotecas y centros de documentación en nuestro país, y averiguar en qué medida la era digital ha propiciado cambios en los centros encuestados.

Los aspectos normativos se presentaron en un artículo anterior publicado en esta misma revista (Gil Urdiciain y Ríos Hilario, 2012), y de los otros dos damos cuenta en el presente trabajo.

El estudio que realizamos se centra en averiguar el tipo de estructura de representación del 
conocimiento utilizado en diferentes bibliotecas, sin entrar a detallar el tipo de preparación de los indizadores/bibliotecarios, ni el nivel de especificidad (cualidad de la indización/clasificación), ya que por una experiencia anterior (Gil Urdiciain, 2005), encontramos dificultades para conseguir respuestas a estas cuestiones.

El objetivo era contactar con el mayor número posible de bibliotecas de titularidad estatal (tanto las bibliotecas públicas autonómicas, como la bibliotecas públicas locales), así como recopilar información procedente de bibliotecas universitarias, tanto públicas como privadas.

Nuestro ideal de consulta hubiera sido la totalidad de centros existentes en España, pero su número (Directorio de bibliotecas españolas, en línea) lo hace inviable para nuestras posibilidades.

\section{Metodología}

Se procedió a la recopilación de datos de tipo directo, mediante preguntas realizadas a través de correo electrónico o in situ, en las propias bibliotecas.

Las preguntas se formularon a los responsables de cada biblioteca y, excepcionalmente, en aquellos casos en los que no se obtuvo respuesta mediante la consulta a través de correo, se realizó la entrevista telefónicamente.

Como herramienta para la consulta se utilizó un cuestionario estructurado con preguntas destinadas a profesionales responsables de bibliotecas y centros de documentación de bibliotecas, tanto de titularidad estatal como de bibliotecas universitarias.

La recogida de datos se realizó durante los meses de marzo y abril de 2011, otorgándose a los encuestados amplio margen de flexibilidad a la hora de responder, dado que las encuestas se realizaron por correo electrónico.

Sin embargo, como suele ocurrir en este método de recogida de datos, el índice de respuesta fue bajo, aunque, como mostramos gráficamente en el apartado de resultados, no en todos los centros se ha dado este nivel de respuesta.

\section{Resultados}

Este apartado hace referencia al índice de respuesta, a los medios de control del vocabulario empleados, y a los cambios que se han producido con la llegada de la era digital, que es uno de los objetivos marcados para este trabajo.

\section{1. Índice de respuesta}

En este apartado mostramos el índice de respuesta y los medios de control del vocabulario empleados en aquellos centros de los que obtuvimos información.

Se presentan los datos relativos a las bibliotecas de titularidad estatal y los obtenidos de las bibliotecas universitarias.

En relación con estas últimas, incluimos en este trabajo los datos recibidos actualmente, limitándonos a aportar aquellos de los centros de los que hemos obtenido respuesta.

En un trabajo anterior (Gil Urdiciain, 2005) incluíamos información de la totalidad de las bibliotecas que conforman la red Rebiun, si bien es cierto que los datos no han variado en el curso de los siete años que los separan.

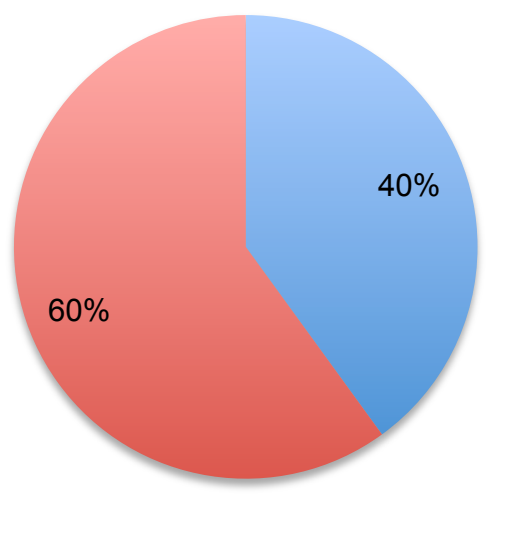

Contestaron No contestaron

Gráfico 1. Índice de respuesta de las bibliotecas estatales

3.2. Uso de sistemas de representación del conocimiento y control del vocabulario

Son mayoría absoluta las bibliotecas estatales que utilizan para la representación del conocimiento la Clasificación Decimal Universal y la Lista de Encabezamientos de Materias para las Bibliotecas Públicas (LEMBP).

En menor medida se emplean listas de encabezamientos propias creadas en base al Catálogo de Autoridades de la Biblioteca Nacional. 


\begin{tabular}{|c|c|c|}
\hline Biblioteca & Sister & as que utilizan \\
\hline Álava & CDU & $\begin{array}{l}\text { Autoridades de materias de la Biblioteca Nacional de España, Lista de } \\
\text { encabezamientos de materia de la Universidad de Sevilla, Lista de encabezamientos } \\
\text { de materia del CSIC }\end{array}$ \\
\hline $\begin{array}{l}\text { Almería Biblioteca } \\
\text { Pública Provincial } \\
\text { "Francisco } \\
\text { Villaespesa" }\end{array}$ & CDU & LEMBP \\
\hline Ávila & CDU & $\begin{array}{l}\text { Catálogo de autoridades de la Biblioteca Nacional, Catálogo de autoridades del CSIC, } \\
\text { Catálogo de autoridades de la Library of Congress }\end{array}$ \\
\hline Calalunya & CDU & $\begin{array}{l}\text { Llista d'encapçalaments de matèria en Català (LEMAC), Clasificación de registros } \\
\text { sonoros de la Xarxa de Biblioteques Populars de la Diputación de Barcelona }\end{array}$ \\
\hline Cádiz & CDU & LEMBP \\
\hline Cantabria & CDU & $\begin{array}{l}\text { LEMBP, Catálogo de Autoridades de materias de la Biblioteca Nacional de España, } \\
\text { Catálogo de Autoridades de la Library of Congress, Catálogo de Autoridades de la } \\
\text { Bibliotèque Nationale de France }\end{array}$ \\
\hline $\begin{array}{l}\text { Castilla y León } \\
\text { (Valladolid) }\end{array}$ & CDU & Catálogo de Autoridades de materias de la Biblioteca Nacional de España \\
\hline $\begin{array}{l}\text { Castilla-La Mancha } \\
\text { (Toledo) }\end{array}$ & CDU & LEMBP \\
\hline Coruña & CDU & LEMBP, Listas elaboradas en Galicia \\
\hline Cuenca & CDU & $\begin{array}{l}\text { LEMBP, Lista de materias para grabaciones sonoras, Lista de descriptores para } \\
\text { literatura (jóvenes y adultos), Listado de descriptores (obras infantiles), Descriptores } \\
\text { para videograbaciones (géneros cinematográficos) }\end{array}$ \\
\hline Gerona & CDU & LEMAC \\
\hline Gijón & CDU & LEMBP \\
\hline Huesca & CDU & \\
\hline Maó & CDU & LEMBP, Llista d'encapçalaments de matèria en català \\
\hline Murcia & CDU & $\begin{array}{l}\text { Autoridades de materias de la Biblioteca Nacional, Géneros cinematográficos de la } \\
\text { Biblioteca Nacional Clasificación Francesa para los registros sonoros }\end{array}$ \\
\hline Palencia & CDU & $\begin{array}{l}\text { Manual de procedimiento para autoridades de la red de bibliotecas de Castilla y León } \\
\text {-que remite al Catálogo de Autoridades de la Biblioteca Nacional-; Música y } \\
\text { grabaciones sonoras no musicales: Manual de procedimiento para autoridades de la } \\
\text { red de bibliotecas de Castilla y León (Términos para música y grabaciones sonoras no } \\
\text { musicales); Videograbaciones: Manual de procedimiento para autoridades de la red } \\
\text { de bibliotecas de Castilla y León; Listado de géneros de cine }\end{array}$ \\
\hline Pontevedra & CDU & $\begin{array}{l}\text { LEMBP; listados propios, elaborados por la Red de biblioteca de Galicia, de géneros } \\
\text { literarios, para asignar encabezamientos de materia a las obras de ficción y de } \\
\text { términos de indización para cine y música. (Se pueden consultar los manuales de } \\
\text { procedimiento en: http://www. rbgalicia. org/index } 2 \text {. php? ap=20\&subApartado=60.) }\end{array}$ \\
\hline $\begin{array}{l}\text { Santa Cruz de } \\
\text { Tenerife }\end{array}$ & CDU & LEMBP \\
\hline $\begin{array}{l}\text { Santiago de } \\
\text { Compostela }\end{array}$ & CDU & Listas propias para diferentes tipos de documentos \\
\hline Soria & CDU & LEMBP, Catálogo de Autoridades de materias de la Biblioteca Nacional de España \\
\hline Zamora & CDU & LEMBP, Catálogo de Autoridades de materias de la Biblioteca Nacional de España \\
\hline
\end{tabular}

Tabla 1: Sistemas de representación del conocimiento en bibliotecas estatales 
Las bibliotecas universitarias utilizan las normas anteriores; e incluyen, básicamente, la Lista de encabezamientos de materia de la Universidad de Sevilla y la Lista de encabezamientos de materia del CSIC.

La Lista d'encapçalaments de matèria en Català (LEMAC) y la Clasificación de registros sonoros de la Xarxa de Biblioteques Populars de la Diputación de Barcelona son de elección para las bibliotecas universitarias de Cataluña.

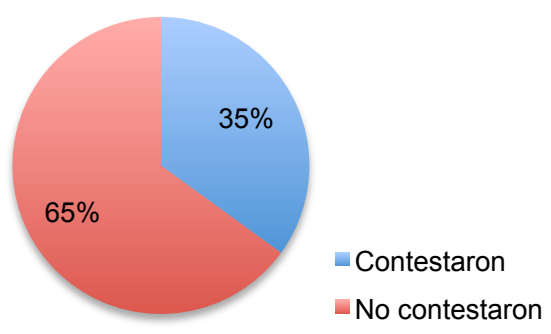

Gráfico 2. Índice de respuesta de las bibliotecas universitarias

\begin{tabular}{lll}
\hline Biblioteca & Sistemas que utilizan & \\
\hline Alcalá de Henares & CDU & Lista de Autoridades (Biblioteca Nacional); CSIC (Catálogo de \\
Servicios & & Autoridades del CSIC); LCSH; CANTIC: Autoridades de nombres y \\
centrales, & títulos de Cataluña; LEMAC: Lista de encabezamientos de materia en \\
Facultad de & catalán; AUBI: Autoridades del servicio de Bibliotecas de Euskadi; \\
Medicina & BNF (Autoridades B. N. Francia); DB (Biblioteca Nacional Alemania); \\
& KVK (Karlsruhe Virtual Katalog, Catálogo colectivo internacional, \\
& sobre todo de bibliotecas de habla alemana); BLIC (British Library \\
& Integrated Catalog); VIAF (Fichero de Autoridades Virtual \\
& Internacional).
\end{tabular}

\begin{tabular}{|c|c|c|}
\hline $\begin{array}{l}\text { Almería } \\
\text { Biblioteca Nicolás } \\
\text { Salmerón de la } \\
\text { Universidad de }\end{array}$ & CDU & $\begin{array}{l}\text { Lista de encabezamientos de materias de la red de bibliotecas del } \\
\text { CSIC, } 4^{a} \text { ed., } 1995 \text {. Además, para algunas materias se emplean } \\
\text { vocabularios más específicos; concretamente, para clasificar los } \\
\text { recursos de Derecho se toman términos del Tesauro de la Biblioteca } \\
\text { de la Facultad de Derecho; en el caso de Psicología, se utiliza el } \\
\text { Tesauro ISOC de Psicología. }\end{array}$ \\
\hline $\begin{array}{l}\text { Universidad de } \\
\text { Castilla La Mancha } \\
\text { (Ciudad Real) }\end{array}$ & CDU & $\begin{array}{l}\text { Autoridades de la BNE; Autoridades del CSIC; Autoridades de la } \\
\text { Library of Congress; Tesauro UNESCO (versión en Castellano } \\
\text { FECYT) }\end{array}$ \\
\hline $\begin{array}{l}\text { Universitat de les } \\
\text { Illes Balears }\end{array}$ & $\begin{array}{l}\text { DDC (Dewey Decimal } \\
\text { Classification) }\end{array}$ & $\begin{array}{l}\text { LEMAC: Llista d'encapçalaments de matèria en Català; LCSH: Library } \\
\text { of Congress Subject Headings }\end{array}$ \\
\hline $\begin{array}{l}\text { Universidad } \\
\text { Autónoma de } \\
\text { Barcelona }\end{array}$ & CDU & $\begin{array}{l}\text { Library of Congress Authorities; Llista d'encapçalaments de matèria } \\
\text { en Català: LEMAC }\end{array}$ \\
\hline $\begin{array}{l}\text { Universidad de } \\
\text { Cantabria }\end{array}$ & $\begin{array}{l}\text { CDU y las siguientes } \\
\text { clasificaciones } \\
\text { especializadas: } \\
\text { AMS (matemáticas), } \\
\text { Inspec (física), } \\
\text { NLM (medicina), } \\
\text { Friburgo (derecho) }\end{array}$ & Lista de encabezamientos de la Universidad de Sevilla \\
\hline Universidad CEU & CDU & Lista de encabezamientos de materia de la UCM \\
\hline $\begin{array}{l}\text { Universidad de } \\
\text { Deusto }\end{array}$ & CDU & $\begin{array}{l}\text { Catálogos de la Biblioteca Nacional; Library of Congress, catálogos } \\
\text { colectivos, etc. Sobre todo, para los conceptos más nuevos de las } \\
\text { diferentes ciencias. }\end{array}$ \\
\hline $\begin{array}{l}\text { Universidad de } \\
\text { Extremadura }\end{array}$ & CDU & Lista de encabezamientos de la Universidad de Sevilla \\
\hline $\begin{array}{l}\text { Biblioteca } \\
\text { Universitaria de } \\
\text { Granada }\end{array}$ & $\begin{array}{l}\text { CDU para la gran } \\
\text { mayoría de las } \\
\text { monografías y los } \\
\text { códigos de la }\end{array}$ & $\begin{array}{l}\text { Lista de encabezamientos de materia para bibliotecas de la OEA. Los } \\
\text { términos nuevos los introducen a partir de los catálogos de bibliotecas } \\
\text { universitarias de temática parecida a la suya (universidades } \\
\text { andaluzas y Universidad Complutense de Madrid). Siguen una } \\
\text { filosofía de descriptores, por lo que no usan encabezamientos y }\end{array}$ \\
\hline
\end{tabular}




\begin{tabular}{|c|c|c|}
\hline & $\begin{array}{l}\text { Unesco para la } \\
\text { producción científica }\end{array}$ & subencabezamientos \\
\hline $\begin{array}{l}\text { Universidad de } \\
\text { Huelva }\end{array}$ & CDU & $\begin{array}{l}\text { Lista de encabezamientos de materias de la Universidad de Sevilla; y } \\
\text { el propio catálogo FAMA (Biblioteca Universitaria de Sevilla), cuando } \\
\text { se necesita la incorporación de un nuevo término como autoridad- } \\
\text { materia. }\end{array}$ \\
\hline $\begin{array}{l}\text { Universidad } \\
\text { Francisco de } \\
\text { Vitoria, Pozuelo de } \\
\text { Alarcón (Madrid) }\end{array}$ & CDU & $\begin{array}{l}\text { Autoridades de la Biblioteca Nacional; Library of Congress Subject } \\
\text { Headings; Lista de autores y entidades del CSIC. }\end{array}$ \\
\hline $\begin{array}{l}\text { Universidad } \\
\text { Camilo José Cela } \\
\text { Villafranca del } \\
\text { Castillo (Madrid) }\end{array}$ & CDU & $\begin{array}{l}\text { Lista de Encabezamientos del CSIC y Encabezamientos de Materia } \\
\text { de la Universidad de Sevilla. }\end{array}$ \\
\hline $\begin{array}{l}\text { Universidad de } \\
\text { Navarra }\end{array}$ & CDU & $\begin{array}{l}\text { Répertoire de vedettes-matière de la Université LavCDU al; Catálogo } \\
\text { de la Biblioteca Nacional de España; Library of Congress Subject } \\
\text { Headings. Actualmente para la asignación de materias se basan en el } \\
\text { listado creado en Millennium. }\end{array}$ \\
\hline $\begin{array}{l}\text { Universidad de } \\
\text { Oviedo }\end{array}$ & CDU & $\begin{array}{l}\text { Library of Congress Subject Headings: Listas de encabezamientos de } \\
\text { la Universidad de Sevilla. Se utilizan de apoyo y orientación } \\
\text { cualquiera de las demás listas de encabezamientos reconocidas: } \\
\text { BNE, CSIC, etc. }\end{array}$ \\
\hline $\begin{array}{l}\text { Biblioteca de la } \\
\text { Universidad de Las } \\
\text { Palmas de Gran } \\
\text { Canaria }\end{array}$ & CDU & $\begin{array}{l}\text { Lista de encabezamientos de materia de la Universidad de Sevilla; } \\
\text { Lista de encabezamientos de materia de la red de bibliotecas del } \\
\text { CSIC. }\end{array}$ \\
\hline $\begin{array}{l}\text { Universidad de } \\
\text { Santiago de } \\
\text { Compostela }\end{array}$ & CDU & Répertoire de vedettes-matière de la Université Laval \\
\hline $\begin{array}{l}\text { Biblioteca de la } \\
\text { UNED }\end{array}$ & CDU & $\begin{array}{l}\text { Una lista de descriptores propia, tipo Tesauro, pero ajustada a las } \\
\text { materias que se imparten en la UNED }\end{array}$ \\
\hline Universitat de Vic & CDU & Llista d'encapçalements de matèria en català (LEMAC) \\
\hline
\end{tabular}

Tabla 2: Sistemas de Representación del Conocimiento en Bibliotecas Universitarias

\subsection{Cambio digital}

En lo que al cambio digital se refiere, es de destacar que, para favorecer la consulta y acceso a los medios digitales, algunas bibliotecas ofrecen programas de alfabetización informacional en línea, con elevados niveles de participación. Las respuestas recibidas en relación con los cambios producidos por la digitalización, muestran las siguientes tendencias:

- Se han producido cambios en las tareas de bibliotecarios y documentalistas, que requieren la actualización de sus conocimientos.

- Las bibliotecas universitarias permiten al usuario, a través del repositorio institucional, el acceso electrónico a los textos completos de gran parte de la producción científica.

- Agilización del préstamo y aumento de posibilidades de recuperar información. En con- secuencia, mayor accesibilidad y utilización de las colecciones.

- Mayor accesibilidad a los contenidos tanto en forma, como en cantidad y en tiempo.

- Posibilidad de realizar renovaciones o reservas desde el opac.

- El impacto de las colecciones electrónicas ha sido determinante, sobre todo por lo que respecta a las publicaciones periódicas.

- En medios audiovisuales, la digitalización permite prestar servicio a los usuarios 24 horas al día, con la doble ventaja de que no se daña el archivo original y resulta barato para la empresa.

- En los últimos cinco años se ha duplicado el número de bases de datos disponibles en algunas bibliotecas. 
- Algunos centros señalan como inconveniente el hecho de que se genera un exceso de documentación que dificulta mucho la selección de la información relevante.

- Posibilidad de ofrecer servicios de mayor calidad que en la era manual.

- Incremento de la posibilidad de compartir recursos.

- Ahorro de espacio, ya que muchos recursos, como por ejemplo las publicaciones periódicas, se suscriben únicamente en formato electrónico.

- Posibilidad del bibliotecario de generar listados, hacer cambios globales, generar estadísticas, etc.

- La opción de integrar los módulos de préstamo, catalogación, revistas y adquisiciones en un mismo sistema facilita mucho el trabajo, porque la información está centralizada en un mismo lugar.

Es significativo que en los datos obtenidos no se muestre una relación entre nuevas formas de comunicación y plataformas con gran cantidad de materiales audiovisuales (como por ejemplo, entre repositorios docentes o de investigación y las bibliotecas universitarias) y una referencia clara a los nuevos sistemas de etiquetado social.

\section{Conclusiones}

Pese a los manifiestos cambios del control del vocabulario, debidos al creciente uso de los documentos digitales en Internet, la representación del conocimiento permanece inalterada en significativas instituciones y no hay perspectivas de cambio en este sentido.

No hay indicios de interoperabilidad entre sistemas, en los centros encuestados; lo que sí existe es el uso combinado de diferentes sistemas. Es común la representación, como ha venido siendo tradicional, mediante listas de encabezamientos de materias y sistemas de clasificación. No obstante, son minoría las bibliotecas que se valen de más de dos sistemas de representación al mismo tiempo.

La Clasificación Decimal Universal (CDU) es el sistema de clasificación más utilizado; le siguen las Listas de Encabezamientos de Materias para las Bibliotecas Públicas; y, por último, tesauros especializados.

El cambio digital ha supuesto mejoras en todos los aspectos: cooperación, normalización y rentabilidad del trabajo; accesibilidad y actualiza- ción de la información y, por supuesto, visibilidad de la biblioteca o centro de documentación.

Se puede igualmente concluir que la digitalización ha propiciado la agilidad de las búsquedas, lo que, en consecuencia, favorece la autonomía del usuario y proporciona mayor interacción entre bibliotecarios/documentalistas y usuarios.

Consideramos de interés y tenemos en proyecto hacer extensiva la consulta a otros centros de documentación y bibliotecas, tanto de carácter público como privado, para conocer el nivel de innovación que han introducido en sus modelos de representación del conocimiento. También nos gustaría ampliar este trabajo para saber hasta qué punto los usuarios encuentran la información adecuada a sus perfiles de búsqueda con la ayuda de los métodos de control del vocabulario disponibles en cada centro.

\section{Notas}

(1) "La nueva ecología de la información y la documentación en la sociedad del conocimiento: desarrollo de una métrica sistémica, planificación de un observatorio para su seguimiento e identificación de tendencias básicas y retos estratégicos". Director: F. J. García Marco. Proyecto I+D+i 2009/07619 del Ministerio de Ciencia e Innovación.

\section{Referencias}

Biblioteca Nacional de España (2012). La difusión de la colección en la era de Google. Madrid: BNE, 2012. http://www.bne.es (2013/07/10).

Directorio de bibliotecas españolas [en línea]. [Madrid]: Ministerio de Educación, Cultura y Deporte, Secretaría de Estado de Cultura, 2012. http://directoriobibliotecas.mcu.es/portada.html/ (2012/06/30).

Gil Urdiciain, Blanca (2005). La organización del conocimiento aplicada a las colecciones universitarias, en Orera Orera, Luisa (edit.), La biblioteca universitaria en su entorno híbrido. Madrid: Síntesis. 287-316.

Ríos Hilario, Ana Belén; Gil Urdiciain, Blanca (2012). Análisis de la normativa en Información y Documentación durante la primera década del siglo $X X I$ (2000-2010). // Scire: Representación y organización del conocimiento. 18: 2, 13-18.

Kristell, Ana. Métodos de recopilación de datos. [S.I. : s.n, 201-?]. http://www.slideshare.net/kriss2505/mtodos-derecoleccin-de-datos (2013/07/10).

Enviado: 2013-01-19. Segunda versión: 2013-07-15. Aceptado: 2013-08-18. 